\title{
Combination Therapy of Alpha-Glucosidase Inhibitor, Thiazolidinedione and Sodium Glucose Co-Transporter-2 Inhibitor in Japanese Type 2 Diabetes Patients
}

\author{
Masataka Kusunoki a, f, Naomi Wakazono ${ }^{\text {a }}$, Kazuhiko Tsutsumi ${ }^{\text {, }}$, \\ Yoshiharu Oshidac ${ }^{c}$, Tesuro Miyata ${ }^{\mathrm{d}, \mathrm{e}}$
}

\begin{abstract}
Background: We additionally administered selective inhibitor of sodium glucose co-transporter-2 (SGLT2) inhibitor to type 2 diabetic patients on $\alpha$-glucosidase inhibitor $(\alpha-\mathrm{GI})$ and/or thiazolidinedione (TZD), to determine whether the multi-drug combination therapy including SGLT2 inhibitor further improved the glycemic control in Japanese patients with diabetes mellitus.
\end{abstract}

Methods: The subjects were divided into four groups. Group I was administered an SGLT2 inhibitor in addition to ongoing treatment using hypoglycemic agents other than $\alpha$-GI and SGLT2 inhibitor. Group II was administered an SGLT2 inhibitor in addition to an $\alpha$-GI. Group III was administered an SGLT2 inhibitor in addition to a TZD. Group IV was administered an SGLT2 inhibitor in addition to an $\alpha$-GI and TZD. The body weight and biochemical parameters were measured before and 1, 2 and 3 years after the start of SGLT2 inhibitor treatment.

Results: In patients receiving $\alpha$-GI or TZD, additional administration of an SGLT2 inhibitor resulted in a reduction of the body weight, body mass index (BMI) and serum uric acid level, but had little effect on the glucose or lipid metabolism. On the other hand, additional administration of SGLT2 inhibitor in patients receiving both $\alpha-\mathrm{GI}$ and TZD (three-drug combination therapy) resulted not only in a reduction of the body weight, BMI and serum uric acid, but also in

Manuscript submitted October 26, 2020, accepted November 19, 2020

Published online December 22, 2020

${ }^{a}$ Research Center of Health, Physical Fitness and Sports, Nagoya University, Furo-cho, Chikusa-ku, Nagoya 464-8601, Japan

${ }^{b}$ Okinaka Memorial Institute for Medical Research, 2-2-2 Toranomon, Minatok-ku, Tokyo 105-8470, Japan

${ }^{c}$ Medical Checkup Center, Minami Seikyo Hospital, 2-204 Minami-odaka, Midori-ku, Nagoya 459-8540, Japan

dVascular Center, Sanno Medical Center, 8-5-35 Akasaka, Minato-ku, Tokyo 107-0052, Japan

eOffice of Medical Education, School of Medicine, International University of Health and Welfare, 4-3 Kozunomori, Narita 286-8686, Japan

fCorresponding Author: Masataka Kusunoki, Research Center of Health, Physical Fitness and Sports, Nagoya University, Furo-cho, Chikusa-ku, Nagoya 464-8601, Japan. Email: info@tonyo.jp

doi: https://doi.org/10.14740/jem708 improvement of the glucose and lipid metabolism, with a reduction of the hemoglobin A1c level and increase of the serum high-density lipoprotein cholesterol level.

Conclusion: The results suggest that three-drug combination therapy with $\alpha$-GI, TZD and SGLT2 inhibitor, each of which has different mechanism of action, is beneficial for the treatment of type 2 diabetes mellitus.

Keywords: Combination therapy; SGLT2 inhibitor; $\alpha$-glucosidase inhibitor; Thiazolidinedione; Hemoglobin A1c; Type 2 diabetes

\section{Introduction}

Concomitant therapy with multiple antidiabetic agents with different mechanisms of action is often used in the treatment of patients with type 2 diabetes mellitus showing insufficient glycemic control with a single agent.

The $\alpha$-glucosidase inhibitors ( $\alpha$-GIs) inhibit small-intestinal $\alpha$-glucosidase to reduce the rate of digestion of carbohydrates and suppress glucose transfer into the blood, resulting in suppression of postprandial blood glucose elevation. The $\alpha$-GIs have the advantage that they are associated with a low risk of hypoglycemia and little increase of the body weight [1, 2].

Thiazolidinediones (TZDs) act as agonists of peroxisome proliferator-activated receptor $\gamma$ (PPAR $\gamma$ ), which is a nuclear transcription factor; activation of PPAR $\gamma$ induces large adipocytes to undergo apoptosis and preadipocytes to differentiate into small adipocytes. These remodeling events decrease the secretion of tumor necrosis factor- $\alpha(\mathrm{TNF}-\alpha)$ due to increased adiponectin secretion by small adipocytes and decreased number of large adipocytes, resulting in a reduction of insulin resistance $[3,4]$.

Sodium glucose co-transporter-2 (SGLT2) is a transporter expressed in the renal proximal tubules and is responsible for the reabsorption of approximately $90 \%$ of the glucose filtered by the glomeruli $[5,6]$. SGLT2 inhibitors are thought to exert hypoglycemic effects by selectively inhibiting SGLT2 and thereby promoting excretion of glucose in the urine [7]. In addition, SGLT2 inhibitors have been reported to have a hypo- 
glycemic effect, to reduce the body weight, to lower the blood pressure and to improve lipid or uric acid metabolism [8]. Due to these pleiotropic effects, SGLT2 inhibitors have been shown to significantly suppress heart failure and cardiovascular death as compared to placebo in patients with high cardiovascular risk [9].

That is, the three types of drugs, $\alpha$-GIs, TZD and SGLT2 inhibitors have different mechanisms of action against hyperglycemia. In this study, we additionally administered one of several SGLT2 inhibitors to patients who were receiving one of two $\alpha$-GIs and/or TZD, to investigate the effect of the multidrug combination regimens on the glycemic control in Japanese patients with type 2 diabetes mellitus.

\section{Materials and Methods}

\section{Study design}

Patients participating in the study were explained the purpose of the study by the physicians in charge, and their informed consents were obtained before their participations. This clinical study was officially registered as an open-label study (ID: UMIN000021584). This study, which was conducted at Akishima Clinic (Nagoya, Japan), was approved by the institutional review committee (approval number: 1-1). This study was conducted in compliance with the ethical standards of the responsible institution on human subjects as well as with the Helsinki Declaration.

A total of 60 type 2 diabetic, Japanese patients (42 males and 18 females) attending Akishima Clinic (Nagoya, Japan) were enrolled in the study. The subjects had a hemoglobin A1c $(\mathrm{HbAlc})$ levels of $6.5 \%$ or more and were receiving treatment with one or more antidiabetic drugs. Diet, physical activity and lifestyle including alcohol consumption and smoking of the subjects were unchanged during the study.

Used drugs were: one of two $\alpha$-GIs: voglibose or miglitol and/or TZD: pioglitazone. One of the five SGLT2 inhibitors was used for the additional administration: luseogliflozin, dapagliflozin, tofogliflozin, empagliflozin or canagliflozin. The SGLT2 inhibitor was administered once a day daily before or after breakfast for 3 years. The dose of each drug is summarized in Table 1.

The patients were allocated to four groups: SGLT2 inhibitor monotherapy treatment group $(n=14$, eight males, six females), $\alpha$-GIs + SGLT2 inhibitor treatment group $(\mathrm{n}=17,11$ males, six females), TZD + SGLT2 inhibitor treatment group $(\mathrm{n}=5$, five males) and $\alpha$-GIs + TZD + SGLT2 inhibitor treatment group $(\mathrm{n}=24,18$ males, six females).

\section{Measurement of the body weight, body mass index (BMI), HbA1c, blood lipids and other biochemical parameters}

The body weight was measured, and blood specimens were collected before and 1, 2 and 3 years after the start of SGLT2 inhibitor treatment. Separated serum specimens for measure-
Table 1. Dose Levels of Drugs

\begin{tabular}{|ll|}
\hline Name of drug & Dose level (mg/day) \\
\hline $\begin{array}{l}\alpha \text {-GI } \\
\text { Voglibose }\end{array}$ & 0.9 \\
Miglitol & 150 \\
TZD & \\
$\quad$ Pioglitazone & 30 \\
SGLT2 inhibitor & \\
Luseogliflozin & 2.5 \\
Dapagliflozin & 5 \\
Tofogliflozin & 20 \\
Empagliflozin & 10 \\
Canagliflozin & 100 \\
\hline
\end{tabular}

a-GI, a-glucosidase inhibitor; TZD, thiazolidinedione; SGLT2, sodium glucose co-transporter-2.

ment of the blood biochemical parameters were stored frozen at $-80{ }^{\circ} \mathrm{C}$ until the measurements. The serum $\mathrm{HbA} 1 \mathrm{c}$, lipids, aspartate aminotransferase (AST), alanine aminotransferase (ALT), $\gamma$-glutamyl transpeptidase $(\gamma$-GTP) and uric acid were measured, using an auto-analyzer (JCA-BM8000 series, JAOL, Tokyo, Japan), by Handa Medical Association Health Center (Aichi, Japan). The HbAlc levels were measured by an automated high-performance liquid chromatography (HPLC) method (HLC-723GX, Tosoh Corporation, Tokyo, Japan).

\section{Statistical analysis}

All the data are expressed as the mean \pm standard deviation (SD). The comparison of pre- vs. post-treatment data was conducted using one-way analysis of variance (ANOVA) (repeated measures) and Dunnett's post hoc test.

\section{Results}

\section{Group treated with antidiabetic agent(s) other than any of a-GIs, TZD or SGLT2 inhibitors}

The body weight, BMI and serum uric acid level decreased at 1 or 2 years after the start of SGLT2 inhibitor treatment. The effects persisted up to the end of 3-year study period. The serum $\mathrm{HbA1}$ c level was not changed throughout the study period (Table 2).

\section{$\alpha-G I$ treatment}

The body weight and BMI were reduced at 1 year, and the effects persisted up to the end of the 3-year study period. The serum $\mathrm{HbA} 1 \mathrm{c}$ level did not change throughout the study period. 





Decrease of the serum ALT was observed at 2 years. Decrease of the serum uric acid was observed at 1 and 2 years. The additional SGLT2 inhibitor treatment had no effects on the blood lipid profile (Table 2).

\section{TZD treatment}

The additional SGLT2 inhibitor treatment had no effects on the body weight, BMI, HbA1c, ALT, $\gamma$-GTP and blood lipid profile.

The serum uric acid level decreased at 1 and 2 years after the start of the SGLT2 inhibitor treatment (Table 2).

\section{$\alpha-G I+T Z D$ treatment}

Decreases of the body weight, BMI and HbAlc were observed at 1 year after the start of the SGLT2 inhibitor treatment, and the effects persisted up to the end of the 3-year period. Decreases of the serum AST and ALT were observed at 2 or 3 years after treatment, and elevation of the serum high-density lipoprotein cholesterol (HDL-C) was observed at 2 and 3 years. The total cholesterol and low-density lipoprotein cholesterol (LDL-C) did not change throughout the study period. Decrease of the serum uric acid was observed at 1 and 3 years after the start of the SGLT2 inhibitor treatment (Table 2).

\section{Discussion}

We additionally administered a selective inhibitor of SGLT2 for 3 years to type 2 diabetic, Japanese patients with stable glycemic control under treatment with $\alpha$-GI and/or TZD, to determine if the additional SGLT2 inhibitor treatment further improved the glycemic control.

In diabetic patients whose blood glucoses were controlled by treatment with hypoglycemic agents other than $\alpha$-GIs, TZD and SGLT2 inhibitors, SGLT2 inhibitor decreased the body weight, BMI and serum uric acid level, while there was no effect on the HbA1c level. SGLT2 inhibitors have been reported to reduce the body weight, BMI and serum uric acid level [8, 10]. The results of the present study suggest that the additional administration of SGLT2 inhibitor has a beneficial effect on the body weight, BMI and serum uric acid. It had little effect on the HbA1c, however, suggesting that the treatment had no beneficial effect on the glucose metabolism. In addition, the treatment had no effect on the blood lipid profile.

In the group of ongoing treatment with $\alpha$-GI, the body weight, BMI and serum uric acid level were observed at the 2or 3-year; on the other hand, the HbA1c level was not reduced. In this group, the addition of the SGLT2 inhibitor could have a beneficial effect on the body weight, BMI and serum uric acid, although its additional effect on glucose metabolism was not observed. Kashiwagi et al reported that additional administration of ipragliflozin, an SGLT2 inhibitor, for 52 weeks reduced the HbA1c and body weight in type 2 diabetic patients show- ing insufficient glycemic control on treatment with $\alpha$-GI (acarbose, voglibose or miglitol) [11]. In the present study however, the HbA1c did not decrease after the start of the SGLT2 inhibitor treatment. Although the reason for the difference remains unknown, the effect of combination therapy with $\alpha$-GI and SGLT2 inhibitor in reducing the HbA1c may take place only after the course of longer-term treatment. Furthermore, study by Kashiwaga et al enrolled patients with insufficient glycemic control; on the other hand, our study enrolled patients with relatively good glycemic control.

In the group with ongoing TZD treatment, the serum uric acid level decreased at the 1 or 2 years after the start of the additional SGLT2 inhibitor treatment, while the body weight, $\mathrm{BMI}$ and HbA1c did not change. Some previous studies reported that additional administration of an SGLT2 inhibitor in patients receiving a TZD was associated with a reduction of the HbA1c level [12-14]. Rosenstock et al reported that additional administration of dapagliflozin (5 or $10 \mathrm{mg} /$ day), an SGLT2 inhibitor, for 4 weeks in patients receiving pioglitazone resulted in a reduction of the HbA1c level [12]. The reason for the difference in the results between the present study and those reported by Rosenstock et al and others remains unknown. However, the patients enrolled in the study by Rosenstock et al had insufficient glycemic control, with HbAlc levels of as high as $8.34-8.40 \%$, whereas the patients enrolled in our study showed relatively stable glycemic control, with a mean $\mathrm{HbAlc}$ level of as low as $6.7 \%$ prior to the start of the SGLT2 inhibitor treatment. Nagata et al reported that once long-term SGLT2 inhibitor treatment nearly normalized the blood glucose levels, no further decrease of the blood glucose levels was noted thereafter [15]. Therefore, since our subjects showed relatively low HbA1c levels, the additional administration of an SGLT2 inhibitor may have been less effective. In the future, it is necessary to elucidate the relationship between the HbA1c levels prior to the start of treatment with an SGLT2 inhibitor and the efficacy of this class of drugs.

TZDs are known to cause fluid retention in the body, thereby inducing edema and increasing the body weight [16]. In the present study, no increase of the body weight was observed in the patients. Kovacs et al also reported that additional treatment with empagliflozin for 24 or 76 weeks reduced the body weight in patients receiving treatment with pioglitazone $[13,14]$. This is may be one of the beneficial effects of the additional SGLT2 inhibitor treatment, and thus concomitant treatment with TZD and SGLT2 inhibitor is considered to be clinically beneficial in suppressing the body weight gain, which is a risk factor for heart disease, associated with treatment with TZD alone.

In the patients with ongoing $\alpha$-GI plus TZD treatment, decreases of all of the body weight, BMI, serum uric acid and HbA1c were observed at 1 year after the start of the additional SGLT2 inhibitor treatment, and this effect persisted until the end of the 3-year study period. In addition, the serum HDL-C also increased at 2 years after the start of the SGLT2 inhibitor treatment until the end of study period. Furthermore, the serum AST and ALT, which are parameters of liver dysfunction, also decreased at 1 and 2 years.

SGLT2 inhibitors improve liver dysfunction [17]. The decreases in the serum AST and ALT observed in the present 
study could express the effects. In the aforementioned group, the decreases in $\mathrm{HbAlc}$, body weight, BMI, serum uric acid, and serum ALT and AST, and the increase in serum HDL-C could indicate the beneficial effects of additional administration of SGLT2 inhibitor. The limitations of this study include the patients who received pharmacotherapy other than $\alpha$-GIs, TZD and SGLT2 inhibitor for the treatment of diabetes mellitus, hyperlipidemia, or hypertension.

In conclusion, the results of this study showed that additional administration of SGLT2 inhibitor in patients receiving $\alpha$-GI resulted in a further decrease of the body weight, BMI and serum uric acid level, but had little effect on the glucose or lipid metabolism. SGLT2 inhibitor administered to patients receiving TZD had no effects on the body weight, BMI, HBA1c, blood lipids and liver dysfunction parameters. On the other hand, additional administration of SGLT2 inhibitor to patients concurrently receiving both of $\alpha$-GI and TZD resulted in further decreases of all of the body weight, BMI, serum uric acid level and HbAlc level, and the effects remained sustained until the end of the 3-year study period. In addition, increase in serum HDL-C and reduction in serum AST and ALT, clearly indicating the beneficial effect of additional administration of an SGLT2 inhibitor. Our results suggested that three-drug combination therapy with $\alpha-G I$, TZD and SGLT2 inhibitor, all of which have different mechanisms of action, is highly effective for the treatment of type 2 diabetes mellitus.

\section{Acknowledgments}

None to declare.

\section{Financial Disclosure}

None to declare.

\section{Conflict of Interest}

MK and NW belong to the division endowed by Taisho Pharmaceutical Co., Ltd.

\section{Informed Consent}

Appropriate IRB approval was obtained prior to the present study.

\section{Author Contributions}

MK contributed to conception and interpretation of the data. $\mathrm{NW}$ and KT performed the statistical analysis. MK, NW, KT, YO and TM were involved in writing, reviewing and editing the manuscript. All the authors read and approved the final manuscript.

\section{Data Availability}

The authors declare that data supporting the findings of this study are available within the article.

\section{Abbreviations}

$\alpha$-GI: $\alpha$-glucosidase inhibitor; ALT: alanine aminotransferase; AST: aspartate aminotransferase; BMI: body mass index; $\gamma$-GTP: $\gamma$-glutamyl transpeptidase; HbA1c: hemoglobin A1c; HDL-C: high-density lipoprotein cholesterol; HPLC: highperformance liquid chromatography; LDL-C: low-density lipoprotein cholesterol; PPAR $\gamma$ : peroxisome proliferator-activated receptor $\gamma$; SGLT2: sodium glucose co-transporter-2; TNF- $\alpha$ : tumor necrosis factor- $\alpha$; TZD: thiazolidinedione

\section{References}

1. Scott LJ, Spencer CM. Miglitol: a review of its therapeutic potential in type 2 diabetes mellitus. Drugs. 2000;59(3):521-549.

2. Standl E, Schnell O. Alpha-glucosidase inhibitors 2012 cardiovascular considerations and trial evaluation. Diab Vasc Dis Res. 2012;9(3):163-169.

3. McGuire DK, Inzucchi SE. New drugs for the treatment of diabetes mellitus: part I: Thiazolidinediones and their evolving cardiovascular implications. Circulation. 2008;117(3):440-449.

4. Sato D, Oda K, Kusunoki M, Nishina A, Takahashi K, Feng Z, Tsutsumi K, et al. PPARgamma activation alters fatty acid composition in adipose triglyceride, in addition to proliferation of small adipocytes, in insulin resistant high-fat fed rats. Eur J Pharmacol. 2016;773:71-77.

5. Wright EM. Glucose transport families SLC5 and SLC50. Mol Aspects Med. 2013;34(2-3):183-196.

6. Wright EM, Loo DD, Hirayama BA. Biology of human sodium glucose transporters. Physiol Rev. 2011;91(2):733794.

7. DeFronzo RA, Davidson JA, Del Prato S. The role of the kidneys in glucose homeostasis: a new path towards normalizing glycaemia. Diabetes Obes Metab. 2012;14(1):514.

8. Seino Y, Sasaki T, Fukatsu A, Ubukata M, Sakai S, Samukawa Y. Efficacy and safety of luseogliflozin as monotherapy in Japanese patients with type 2 diabetes mellitus: a randomized, double-blind, placebo-controlled, phase 3 study. Curr Med Res Opin. 2014;30(7):12451255 .

9. Zinman B, Wanner C, Lachin JM, Fitchett D, Bluhmki E, Hantel S, Mattheus M, et al. Empagliflozin, cardiovascular outcomes, and mortality in type 2 diabetes. N Engl J Med. 2015;373(22):2117-2128.

10. Kusunoki M, Natsume Y, Miyata T, Tsutsumi K, Oshida Y. Effects of Concomitant Administration of sodium glucose co-transporter 2 inhibitor with insulin on hemoglobin alc, body mass index and serum lipid profile 
in Japanese type 2 diabetic patients. Drug Res (Stuttg). 2018;68(12):669-672.

11. Kashiwagi A, Isaka H, Takinami Y, Kazuta K, Utsuno A. Long-term safety, tolerability and efficacy of ipragliflozin in combination with an $\alpha$-glucosidase inhibitor in Japanese patients with type 2 diabetes mellitus inadequately controlled with an $\alpha$-glucosidase inhibitor alone. Jpn Pharmacol Ther. 2014;42:923-939.

12. Rosenstock J, Vico M, Wei L, Salsali A, List JF. Effects of dapagliflozin, an SGLT2 inhibitor, on $\mathrm{HbA}(1 \mathrm{c})$, body weight, and hypoglycemia risk in patients with type 2 diabetes inadequately controlled on pioglitazone monotherapy. Diabetes Care. 2012;35(7):1473-1478.

13. Kovacs CS, Seshiah V, Merker L, Christiansen AV, Roux F, Salsali A, Kim G, et al. Empagliflozin as add-on therapy to pioglitazone with or without metformin in patients with type 2 diabetes mellitus. Clin Ther. 2015;37(8):1773$1788 \mathrm{e} 1771$.

14. Kovacs CS, Seshiah V, Swallow R, Jones R, Rattunde H, Woerle HJ, Broedl UC, et al. Empagliflozin improves gly- caemic and weight control as add-on therapy to pioglitazone or pioglitazone plus metformin in patients with type 2 diabetes: a 24-week, randomized, placebo-controlled trial. Diabetes Obes Metab. 2014;16(2):147-158.

15. Nagata T, Fukazawa M, Honda K, Yata T, Kawai M, Yamane M, Murao N, et al. Selective SGLT2 inhibition by tofogliflozin reduces renal glucose reabsorption under hyperglycemic but not under hypo- or euglycemic conditions in rats. Am J Physiol Endocrinol Metab. 2013;304(4):E414423.

16. Endo Y, Suzuki M, Yamada H, Horita S, Kunimi M, Yamazaki O, Shirai A, et al. Thiazolidinediones enhance sodium-coupled bicarbonate absorption from renal proximal tubules via PPARgamma-dependent nongenomic signaling. Cell Metab. 2011;13(5):550-561.

17. Kusunoki M, Natsume Y, Sato D, Tsutsui H, Miyata T, Tsutsumi K, Suga T, et al. Luseogliflozin, A sodium glucose Co-transporter 2 inhibitor, alleviates hepatic impairment in Japanese patients with type 2 diabetes. Drug Res (Stuttg). 2016;66(11):603-606. 